\title{
The Advantages of Two Dimensional Techniques (2D) in Pituitary Adenoma Treatment
}

\author{
Diabate Adama ${ }^{1}$, MEM Gar-elnabi ${ }^{2}$, and Mohammed, A. AliOmer ${ }^{2,3}$ \\ ${ }^{1}$ University of Hail, College of Applied Medical Science-Hail, KSA \\ ${ }^{2}$ Sudan University of Science \& Technology, College of Medical Radiological Science, Khartoum - Sudan \\ ${ }^{2,3}$ Qassim University, College of Applied Medical Science, - Buraidah, KSA
}

\begin{abstract}
The purpose of the study is to evaluate the two dimensional dose distribution techniques in pituitary adenoma patient treatment in order to provide $2 D$ dose coverage to the target volume while sparing organs at risk (OARs). The CT simulator was used to radiograph 300 patients of pituitary adenomas to conform $2 D$ dose distribution planning inside the tumour bed, and its structures were delineated; including gross target volume $(G T V)$, clinical target volume (CTV), and planning target volume (PTV)], as well as organs at risks (OARs). Dose distribution analysis was edited to provide $2 D$ dose coverage to the target while sparing organs at risk. The main results of the study were, $2 D$ dose distribution plans increases the unnecessary dose to the critical organs according to their geographical location from the pituitary adenoma site, and the present study, concludes that when the tumour dose increases from 45 to 55 Gy there is a linear proportional increment of dose to the organs at risks, and when the dose is about $60 \mathrm{~Gy}$ in $2 \mathrm{D}$, the increment of unnecessary dose to temporal lobe is $0.31 \mathrm{~Gy}$, and to eye is $0.34 \mathrm{~Gy}$, and to optic chiasm is $0.42 \mathrm{~Gy}$ respectively.New techniques, which will lessen the unnecessary dose to OARs, needed to be developed .
\end{abstract}

Keywords: $2 D$ dose distribution, pituitary adenoma, organ at risks, clinical target volume, planning target volume.

\section{Introduction}

The pituitary gland is the maestro gland of the body system and it controls the activities of other glands in the body situated in sella turcica, and it is related to the critical organs of the brain such as: optic chiasma, temporal lobe, and eye. The pituitary gland has three parts, anterior, posterior, and intermediate lobes. In general pituitary adenoma can be treated with (2D) dose distribution which has a function of two coordination of the depth (axis X) and the width along scan direction (axis $\mathrm{Y}$ ) for each product to be treated in the irradiation facility, because2D technique is a method using in radiation therapy for cancer treatment; in which dose distributions are spatially represented by a source of radiation; therefore this describes the variation of dose within an irradiated target volume ${ }^{(1)}$.

On the other hand, traditional isodose curve is a $2 \mathrm{D}$ representation of dose variation with position and within a beam along directions of both parallel and perpendicular to the beam's direction ${ }^{(2)}$. However, two dimension techniques were associated with an increased risk of locoregional recurrence ${ }^{(3)}$. But $2 \mathrm{D}$ technique is also used for post-operative lesions at high risk, recurrences. The delineation of target volume in 2D is critical, due to the proximity of organs at risk and a risk of late toxicity ${ }^{(4)}$. Therefore, a two dimension margin can then be added to the resulting outline, so as to account for microscopic tumour spread, organ motion, and setup uncertainty ${ }^{(5)}$. The two dimensional (2D) planning dose distribution calculations are faster and easier and can be performed by hand moreover is much less accurate ${ }^{(6)}$.

The 2D technique used in head and neck influences early and late complications ${ }^{(7)}$. This study will add a valuable method for clinical implementation of $2 \mathrm{D}$ techniques in the treatment of pituitary adenoma; and the result of this study the radiotherapy centers will look into this technique for clinical implication and open a further research in order to seek for a better treatment method for pituitary adenoma patients. The main objectives of this study are to evaluate the 2D dose distributions in both tumour bed and surrounding normal tissues, because treatment plan attempts dose distribution to be optimized for a clinical goal in a clinical situation. Meanwhile the objective of radiotherapy is to treat patient with the best therapeutic ratio, in order to achieve highest local control and lowest toxicity to normal tissues.

\section{Materials And Methods}

The irradiation of treatment area control remains a significant problem in management of pituitary tumour, but the dose distribution of 2D in pituitary tumour will eventually provide an answer to the problem in management of pituitary tumour with the ability to deliver a dose to the target volume. Therefore, the target volume should be covered by the $95 \%$ isodose; wherever, required and achievable, the minimum doses to critical organs without compromising the PTV coverage of at least $95 \%$ dose to $95 \%$ of PTV volume ${ }^{(8)}$. 
Furthermore, satisfactory CTV dosimetric criteria were selected to be a minimum CTV dose of $95 \%$ of the PTV dose and at least $95 \%$ of the CTV receiving $100 \%$ of the PTV dose ${ }^{(9)}$.The total tumour dose for pituitary adenomas is 45 and $50 \mathrm{~Gy}$ because these doses are below the tolerance of central nervous system neural tissue ${ }^{(10)}$. The CT scans can provide sufficient discoveries of the pituitary adenoma size ${ }^{(11)}$. Pituitary microadenomas appear lucent on computed tomographic (CT) images ${ }^{(12)}$.

\section{II.1 Population and Sample}

The study sample represents 300 cases of pituitary adenoma with the mean and standard deviation (35 \pm 2.32 respectively). Meanwhile, the samples of the study after the data collection were (female 178, and male 122).

Table .1 pituitary adenoma clinical correlation

\begin{tabular}{|c|c|c|c|c|}
\hline \multirow{2}{*}{ AGE } & \multicolumn{2}{|c|}{ SEX } & \multirow{2}{*}{ MEAN } & \multirow{2}{*}{ SD \pm} \\
\cline { 2 - 3 } & M & F & & 3.7 \\
\hline $10 \leq 19$ & 10 & 18 & 33.4 & 5.8 \\
\hline $20 \leq 29$ & 29 & 36 & 48.2 & 4.9 \\
\hline $30 \leq 39$ & 32 & 54 & 71 & 4.7 \\
\hline $40 \leq 49$ & 31 & 58 & 90 & 4.4 \\
\hline $50 \leq 59$ & 20 & 12 & 107.6 & \\
\hline TOTAL & 122 & 178 & & \\
\hline
\end{tabular}

\section{II.2 Instrumentation}

The CT simulator was used to radiograph case of pituitary adenoma; all patients were CT simulated after properly positioning and immobilization for $2 \mathrm{D}$ plans inside the pituitary adenoma, and its structures were delineated; including the target volume, gross target volume (GTV), clinical target volume (CTV), and planning target volume (PTV), as well as organs at risk. Dose distribution analysis was also edited to provide 2D dose coverage to the target while sparing organs at risk. Plans were later evaluated and compare the dose distributions between PTV dose coverage to pituitary adenoma and OARs. And finally, the treatment was done with high energy Linear Accelerator Clinac 2300 CD Varian having 120 leaf millennium MLC was used for the delivery of treatments.

\section{Results}

The report of all references that we found to each dose limit in figures and tables, regardless of whether each author actually used the dose tolerance limit or not. These findings will deal with 2D dose distribution utilities in radiation therapy in which it has variables such as: temporal lobe, eye, optic chiasma. Then, the results are represented in a form of tables, and histograms.

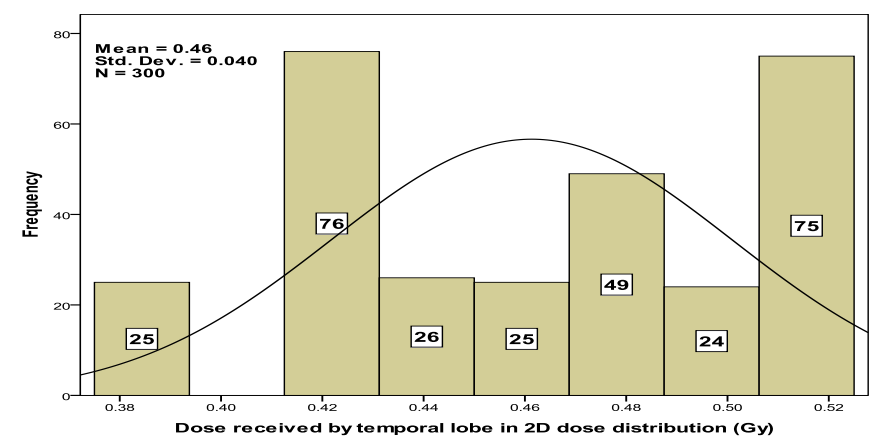

Figure 3.1 (a) (a bar or histogram) shows the frequency distribution of the pituitary adenoma based on dose received by temporal lobe in $2 \mathrm{D}$ dose distribution.

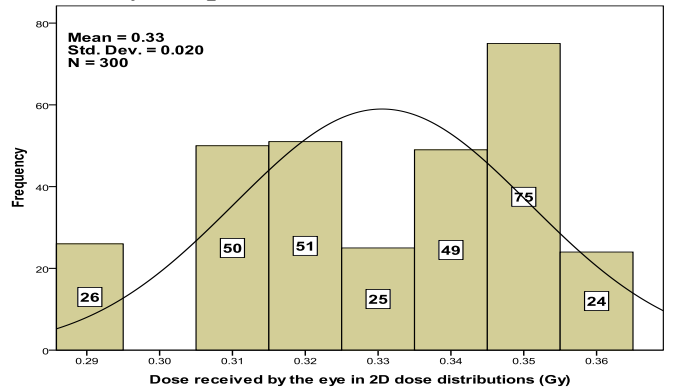

Figure 3.2(b) (a bar or histogram) shows the frequency distribution of the pituitary adenoma based on dose received by eye in $2 \mathrm{D}$ dose distribution. 


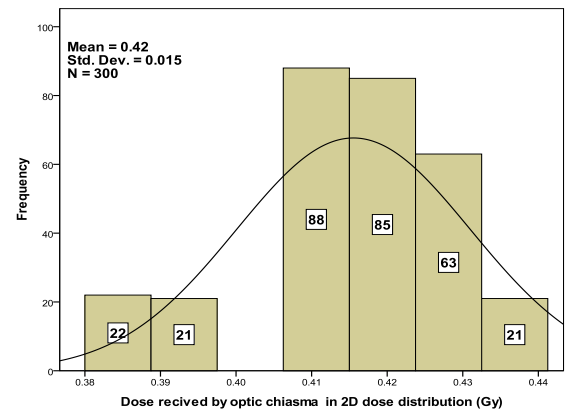

Figure 3.3(c) (a bar or histogram) shows the frequency distribution of the pituitary adenoma based on dose received by optic chiasma in $2 \mathrm{D}$ dose distribution.

In the Fig. $(a, b$, and $c)$ mentioned above are plotted to show the distributions of patient's dose received by temporal lobe, eye, and optic chiasm in 2D dose distribution ; with a normal distribution curve over plotted as well as the mean and standard deviation. The results mainly confirm 2D dose distribution exposes the critical organs to higher doses of radiation, regardless to the variability of the tumour dose, pituitary size, and tumour sizes. Furthermore, two dimension images set accurate target volume delineation to minimize uncertainties that may affect the treatment ${ }^{(13)}$.

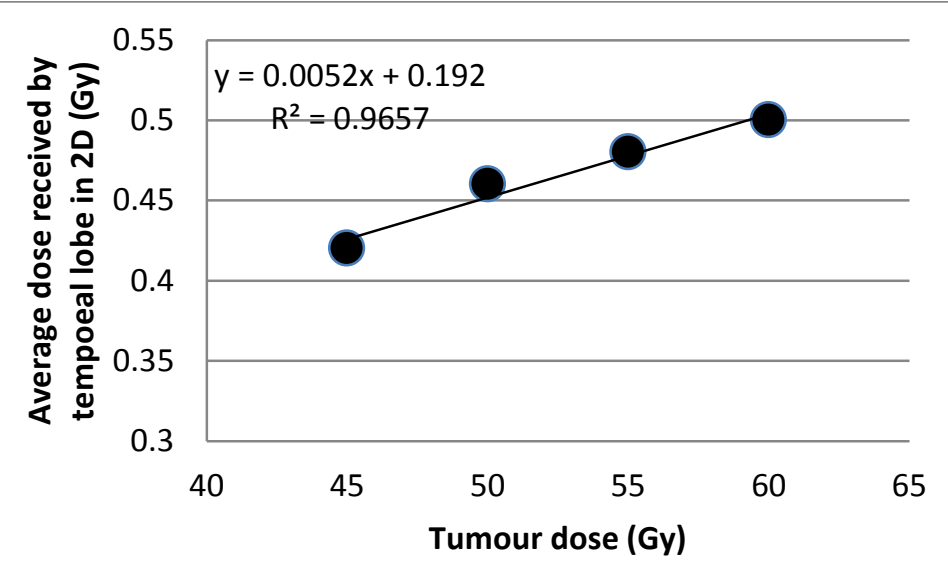

Figure 3-4 (d) scatter plot shows a direct linear relationship between the doses received by eye versus tumor dose using 2D dose distribution.

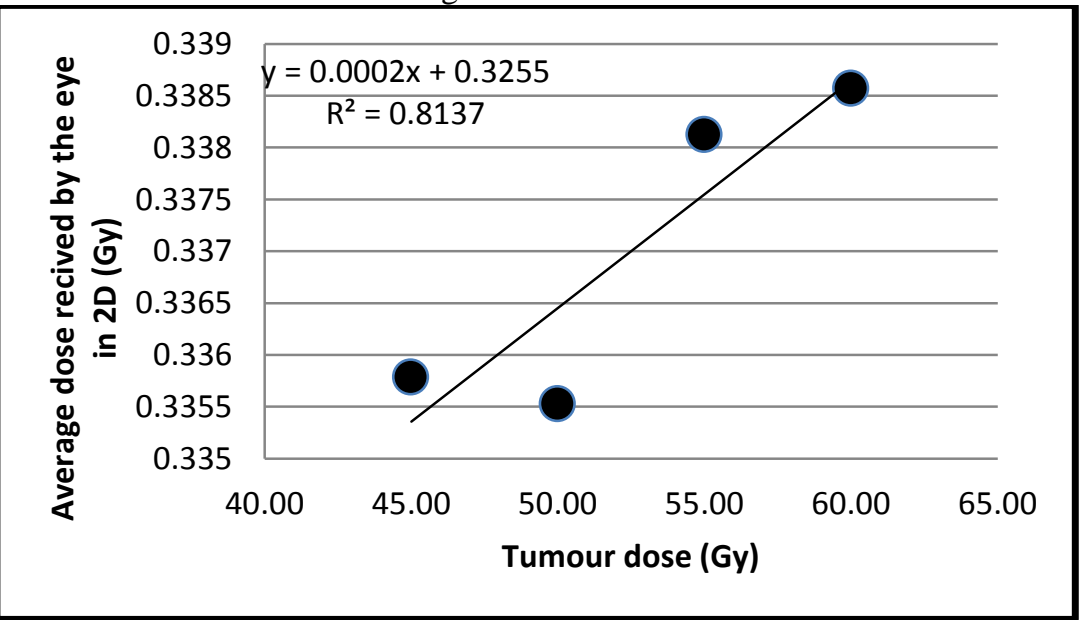

Figure 3-5 (e) scatter plot shows a direct linear relationship between the doses received by eye versus tumor dose using $2 \mathrm{D}$ dose distribution 


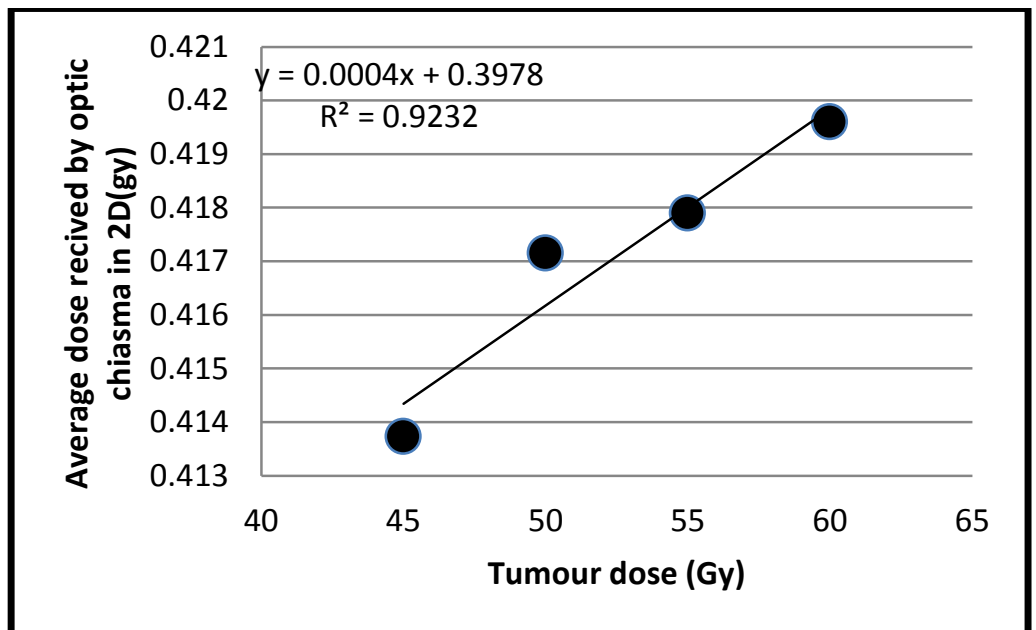

Figure 3-6 (f) scatter plot shows a direct linear relationship between the doses received by optic chiasma versus tumor dose using 2D dose distribution.

In the Fig. (d, e, and f) mentioned above portrayed scatter plot between the tumour dose and the average dose received by temporal lobe, eye and optic chaisma in case of 2D dose distributions; the trend line demonstrate that there is a linear relationship between the tumor dose and the doses received by the critical organs, with a strong association judged by the adjustment of $\mathrm{R}^{2}$.however, there is a relationship between the average tumour sizes, and tumour dose where that shows a direct linear association; which explain the variability in tumour dose.

\section{III.1 Temporal Lobe}

The dose received by temporal lobe in average was $0.46 \pm 0.04 \mathrm{~Gy}$ using $2 \mathrm{D}$ dose distributions, then , the results show that the dose received by temporal lobe was higher when the $2 \mathrm{D}$ distribution; this increment was obvious in respect to tumor dose where the dose in temporal lobe is linearly increased by $0.0052 \mathrm{~Gy}$ per unit of a tumor dose starting $0.2 \mathrm{~Gy}$ as shown in figure $4.4(\mathrm{~d})$. Therefore this result proves that $2 \mathrm{D}$ dose distribution will increase the amount of radiation received by temporal lobe substantially, whenever the total tumour dose increases from $45 \mathrm{~Gy}$ to $60 \mathrm{~Gy}$; but in case of $60 \mathrm{~Gy}$ the dose for temporal lobe increases to $0.31 \mathrm{~Gy}$ for 2D. In particular, , 2D dose distribution technique in the radiation therapy treatment induces temporal lobe necrosis (TLN) hence becomes a common late damage that adversely affects the quality of life ${ }^{(14)}$. However, the hearing loss and facial weakness is a function of cranial nerve damage due to radiation dose escalation to the surrounding tissues ${ }^{(15)}$. Nevertheless, temporal lobe contains rich networks of other sensitive structures that are at risk after radiotherapy and that may contribute to toxicity afterward. Therefore, this result dictates the following: since the tumour dose ranges from 45 to $60 \mathrm{~Gy}, 2 \mathrm{D}$ technique is not an ideal treatment for pituitary adenoma, because it does not prevent unnecessary dose to adjacent tissues. In addition, these findings can be used clinically in order to find more suitable and safe for a pituitary adenoma patients treatment while sparing surrounding normal tissues with unnecessary radiation ${ }^{(16)}$.

\section{III.2 Eye}

Concerning the dose received by eye in average was $0.3 \pm 0.02$ Gy using $2 \mathrm{D}$ dose distributions, then the results show that the dose received by eye was higher in 2D distribution; this increment was obvious in respect to tumor dose where the dose in eye is linearly increased by $0.0003 \mathrm{~Gy}$ per unit of the tumor dose starting from $0.3 \mathrm{~Gy}$ as shown in Figure 4.5(e). Therefore, this result also proves that $2 \mathrm{D}$ dose distribution will not reduce the amount of radiation to the eye substantially, even if the total tumour dose increases from $45 \mathrm{~Gy}$ to $60 \mathrm{~Gy}$, but in case of $60 \mathrm{~Gy}$ the unnecessary dose reaching the eye will be $0.34 \mathrm{~Gy}$ for 2D. In addition, , the risk of central nervous system toxicity to doses less than $50 \mathrm{~Gy}$ at $2 \mathrm{~Gy}$ per fraction is low, with a reported incidence of optic neuropathy resulting in visual deficits of $1-5 \%$, and a risk of necrosis of normal brain structures of $0-2 \%{ }^{(17)}$. However, visual loss is associated with a mass lesion; the cause may interfere with the function of the optic apparatus. Generally the dose reaching the eye should be less than $2 \mathrm{~Gy}$. Thus, 2D techniques do not accomplish the aim of avoiding the eye with unnecessary radiation. These findings can be used in order to seek for better alternative treatment modalities for pituitary adenoma which will avoid the surrounding tissues from unnecessary radiation to the eye glob $^{(18)}$. 


\section{III.3 Optic Chiasma}

The study findings shows that, the dose received by optic chiasma in average was $0.42 \pm 0.015 \mathrm{~Gy}$ when using 2D dose distributions, and this results show that the dose received by optic chiasm was higher if 2Ddose distribution is used; this increment was obvious in respect to tumor dose when the dose in optic chiasma is linearly increased about $0.0002 \mathrm{~Gy}$ per unit of tumour dose starting from $0.2 \mathrm{~Gy}$ as shown in 4.6 (f). Therefore, this result proves that $2 \mathrm{D}$ dose distribution will not reduce the amount of radiation to the optic chiasma substantially even if the total tumour dose increases from $45 \mathrm{~Gy}$ to $60 \mathrm{~Gy}$, where in case of $60 \mathrm{~Gy}$ the dose for optic chiasma was $0.4196 \mathrm{~Gy}$ for $2 \mathrm{D}$ dose distribution. Therefore, radiation toxicity in the optic chiasm attributed to dose received by the optic chiasma where the risk of toxicity is markedly increased at doses less than $60 \mathrm{~Gy}$ at $1.8 \mathrm{~Gy} /$ fraction as well as at less than $12 \mathrm{~Gy}$ for single-fraction ${ }^{(19)}$. The evidence is strong that radiation tolerance is increased with a reduction in the dose per fraction. While, the maximum radiation dose to the optic nerve was $10 \mathrm{~Gy}$ range from 0.4 to $16.0 \mathrm{~Gy}^{(20)}$. However, the $2 \mathrm{D}$ planning dose distribution calculation is faster and easier and can be performed by hand moreover is much less accurate. Meanwhile, , radiation toxicity of the optic nerves and chiasm to dose and dose-volume measures was markedly increased at doses higher than $60 \mathrm{~Gy}$. The strong evidence shows that radiation tolerance increases with a reduction in dose per fraction. These findings can be used in order to seek for better alternative treatment modalities for pituitary adenoma which will avoid the surrounding tissues from unnecessary radiation reaching the optic chiasma ${ }^{(21)}$.

\section{Conclusion}

In this study, it is concluded that the current method of pituitary adenoma treatment by means of 2D dose distribution plan is not adequate from preventing surrounding tissues to unnecessary dose. New technique of dose distribution for $2 \mathrm{D}$ that will cover the pituitary adenoma cases in treatment without reaching radiation to organs at risk (OARs) needs to be developed.

\section{Acknowledgments}

This work is dedicated to the people who are persevering in the fight of their lives against pituitary adenoma. When the doctors say "it's over" that doesn't always mean it is over! For any patient who is determined to continue the fight against cancer, we are committed to providing every reasonable option of treatment technique available, with the best level of precision, skill and knowledge humanly possible during the treatment of pituitary adenoma patients.

\section{References}

[1]. Kaluska, V.T. Lazurik, V.M. Lazurik, G.F. Popov, Yu.V. Rogov, Zimek, Z., Basic laws of boundaries effects for the absorbed dose distribution of electrons in the heterogeneous materials, Journal of Kharkiv University , 6(19) , 2004, 87-50.

[2]. Khan FM: the physics of radiation therapy, ed 2, Baltimore, 1994, Williams \& Wilkins.

[3]. Zhang F; Zheng, M., Dosimetric evaluation of conventional radiotherapy, three dimensional conformal radiotherapy and direct machine parameter optimization intensity-modulated radiotherapy for breast cancer after conservative surgery, Journal of Medical Imaging and Radiation Oncology, 55 (6), 2011, 595-602

[4]. Chand Fouché ME, Colin P, Bondiau PY., Pituitary adenomas: multimodal management and modern irradiation techniques, Cancer Radiother, 1.1, 2012, 90-100.

[5]. Jason G, Volumetric 3d display for radiation therapy planning, Journal of Display Technology, 4 (4), 2008, 23-25.

[6]. Purdy, JA. Dose to normal tissues outside the radiation therapy patient's Treated volume: a review of different radiation therapy techniques, Health Phys,.95 (5), 2008, 666-76.

[7]. Feuvret L, Noël G, Mazeron J, Bey P. (2006).Conformity index: a review,Int J RadiatOncolBiolPhys, 64 (2), 2006, 333-42.

[8]. Hitchen CJ, Osa EO, Dewyngaert JK, Chang J, Narayana A,Dose to craniofacial region through portal imaging of pediatric brain tumour , J ApplClin Med Phys, 13 (1 ), 2012, 3385.

[9]. Brent C. Parker, Almon S. Shiu, Moshe H. Maor, Frederick F. Lang, H. Helen Liu,i R. Allen White, and. Antolak.,John, A., PTV margin determination in conformal SRT of intracranial lesions, Journal of Applied Clinical Medical Physics, 3 (3), 2002,34-36

[10]. Michalski JM, Purdy, JA. Innovations in three-dimensional treatment planning and quality assurance, Tumori, 84 (2), 1998, 127-39.

[11]. Peyster RG, Hoover ED, Adler LP, CT of the normal pituitary stalk, AJNR Am J Neuroradiol, 5(1), 1984, 45-7.

[12]. Roppolo HM, Latchaw RE, Normal pituitary gland: 2. Microscopic anatomy-CT correlation, AJNR Am J Neuroradiol,4(4), $1983,937-44$.

[13]. Joshi R. C., PK Julka, R. Prabhakar, T. K. Rath, Monica Malik, Ganesh, G "Quantification of shift in isocenter for 2-dimensional and 3-dimensional radiotherapy plans for various treatment sites". Internet Journal of Oncology, Vol .56, 2007, pp.67-70

[14]. Sheng-Fa Su, Shao-Ming Huang, Fei Han, Ying Huang, Chun-Yan Chen, Wei-Wei Xiao, Xue-Ming Sun andTai-Xiang Lu ,Analysis of dosimetric factors associated with temporal lobe necrosis (TLN) in patients with nasopharyngeal carcinoma (NPC) after intensity modulated radiotherapy, Radiation Oncology, 8 (17), 2013, 748-717.

[15]. Chen J, Dassarath M, Yin Z, Liu H, Yang K, Wu G,Radiation-induced temporal lobe necrosis in patients with nasopharyngeal carcinoma: a review of new avenues in its management,RadiatOncol, 6 (128), 2011,6-128.

[16]. Linskey ME, Johnstone PA, Radiation tolerance of normal temporal bone structures: implications for gamma knife stereotactic radiosurgery,Int J RadiatOncolBiolPhys, 57 (1), 2003, 196-200.

[17]. Minniti et al. Radiation Oncology, viewed on 2011, 6/:11, and accessedon http://www.ro-journal.com/content/6/1/167.

[18]. Jonathan A. Borden, Treatment of Tumour involving the Optic Nerves and Chiasm, viewed on 7/3/2013, accessed onhttp://www.jonathanborden-md.com/OpticTumour.html.

[19]. Charles Mayo, Mary K. Martel, , Lawrence B. Marks., John Flickinger, Jiho Nam, John Kirkpatrick, Radiation Dose-Volume Effects of Optic Nerves and Chiasm Next, International Journal of Radiation Oncology, Biology Physics, 76 (3), 2010, 28-35 
[20]. Stafford SL, Pollock BE, Leavitt JA, Foote RL, Brown PD, Link MJ,Gorman DA, Tao Y, Lian D, Hui-juan Z, Hui P, Zi-meng J 2011, 'Value of brain magneticresonance imaging and tumor markers in the diagnosis and treatment of intracranial germinoma in children', Zhongguo Yi ХиеКеХиеҮиапХиеВао, 33 (2),2011, 111-5.

[21]. ICRU Reports 50 and 62', Recommendations, viewed on 9 November 2012, and accessed on http://ozradonc.wikidot.com/current-icru- 